\title{
EFFECT OF COLD AND HEAT THERAPIES ON PAIN RELIEF IN PATIENTS WITH DELAYED ONSET MUSCLE SORENESS: A NETWORK META-ANALYSIS
}

Yutan WANG, BS ${ }^{1 \#}$, Hongmei LU, BS ${ }^{2 \#}$, Sijun LI, BS ${ }^{1}$, Yuanyuan ZHANG, BS ${ }^{1}$, Fanghong YAN, MS ${ }^{1}$, Yanan HUANG, $\mathrm{BS}^{1}$, Xiaoli CHEN, BS ${ }^{1,3}$, Ailing YANG, $\mathrm{MS}^{4}$, Lin HAN, $\mathrm{PhD}^{1,3 *}$ and Yuxia MA, MS ${ }^{1 *}$

From the ${ }^{1}$ Evidence-Based Nursing Center, School of Nursing of Lanzhou University, Lanzhou, ${ }^{2}$ Birthing Center, Gansu Provincial Maternity and Child-care Hospital, ${ }^{3}$ Gansu Provincial People's Hospital and ${ }^{4}$ Department of Pediatric Surgery, Second Hospital of Lanzhou University, Lanzhou, China

\#Those authors contributed equally to this work and should be considered co-first authors, *These authors made equal contributions to this work and should be considered co-corresponding authors.

Objective: To comprehensively compare the effectiveness of cold and heat therapies for delayed onset muscle soreness using network meta-analysis.

Methods: Eight Chinese and English databases were searched from date of establishment of the database to 31 May 2021. Cochrane risk-of-bias tool was used to analyse the included randomized controlled trials. Potential papers were screened for eligibility, and data were extracted by 2 independent researchers. Results: A total of 59 studies involving 1,367 patients were eligible for this study. Ten interventions were examined: contrast water therapy, phase change material, the novel modality of cryotherapy, coldwater immersion, hot/warm-water immersion, cold pack, hot pack, ice massage, ultrasound, and passive recovery. Network meta-analysis results showed that: (i) within $24 \mathrm{~h}$ after exercise, hot pack was the most effective for pain relief, followed by contrast water therapy; (ii) within $\mathbf{4 8} \mathrm{h}$, the ranking was hot pack, followed by the novel modality of cryotherapy; and (iii) over $48 \mathrm{~h}$ post-exercise, the effect of the novel modality of cryotherapy ranked first.

Conclusion: Due to the limited quality of the included studies, further well-designed research is needed to draw firm conclusions about the effectiveness of cold and heat therapies for delayed onset muscle soreness.

Key words: network meta-analysis; cold therapy; heat therapy; delayed onset muscle soreness.

Accepted Sep 7, 2021; Epub ahead of print Oct 12, 2021

J Rehabil Med 2022; 54: jrm00258

Correspondence address: Yuxia Ma and Lin Han, Evidence-Based Nursing Center, School of Nursing, University of Lanzhou, No. 28, Yanxi Road, Lanzhou, 730030, Gansu Province, China. E-mail: yuxiama@Izu. edu.cn and 250630258@qq.com

$\mathrm{D}$ elayed onset muscle soreness (DOMS) is the sensation of pain and discomfort in the muscles, often after taking part in unaccustomed physical activity or high-force muscle work, which normally increases in intensity in the first 24-48 h after exercise and peaks at $24-72 \mathrm{~h}$, then lessens, resolving by 5-7 days postexercise (1). DOMS is a common myogenic condition, probably due to pathophysiological changes within the tissue resulting from micro-injuries (2). Although most

\section{LAY ABSTRACT}

The effects of different methods of cold and heat therapy on pain in patients with delayed onset muscle soreness are debated, and there is uncertainty regarding the most effective of these therapies. The aim of this study was to evaluate the effects of different cold and heat treatments on pain in patients with delayed onset muscle soreness. Using network meta-analysis and ranking, it was found that, within $48 \mathrm{~h}$ post-exercise, use of hot-pack was superior to other interventions, whereas, over $48 \mathrm{~h}$ post-exercise, cryotherapy was the optimal intervention for pain relief in patients with delayed onset muscle soreness.

people will experience DOMS at some time, many accept that it is a self-limiting entity, and most do not seek medical or physiotherapeutic intervention. However, Lightfoot et al. (3) state that DOMS can be detrimental to exercise adherence and may have a drastic effect on performance. The symptoms can range from muscle tenderness to severe debilitating pain, which can reduce patients' performance (a reduction in joint range of motion, peak torque, mobility and flexibility, etc.) or seriously affect quality of life $(1,4)$.

Although the mechanisms and treatment strategies remain uncertain, various mechanisms that contribute to DOMS have been adopted, such as muscle spasm, lactic acid accumulation, injury to the muscles and connective tissues. The main bases supporting this theory (muscle connective tissue damage) are as follows: lower oxygen consumption and energy consumption during exercise, but more serious injuries and soreness, and damage to muscle fibres can be seen under the microscope. In addition to serum myoglobin, phosphokinase, trimethylhistidine and hydroxyproline are increased after exercise (5). According to the inflammatory response theory, DOMS is considered to be a series of inflammatory reactions caused by mechanical injury, and $\mathrm{Ca} 2+$ plays a triggering role in the process of muscle soreness. Some inflammatory mediators are needed to produce pain in patients with DOMS, and prostaglandins are the most important inflammatory mediators. The increased concentration of leucolysin and $\mathrm{Ca} 2+$ after exercise can stimulate 
the synthesis of local prostaglandins (5). Therefore, according to these theoretical mechanisms, various treatments for DOMS are available, including nonsteroidal anti-inflammatory drugs (NSAIDs), heat and cold therapy, stretching, transcutaneous electrical nerve stimulation (TENS), rest, etc. These therapies help to promote the recovery of muscle function, decrease the inflammatory response and alleviate the symptoms of DOMS $(6,7)$. It is worth noting that, among these, heat and cold therapy have become very popular, as they are low-cost and simple techniques that can be performed easily in different situations and can be applied by non-medical personnel (e.g. sports and fitness coaches). In addition, previous studies have reported the effectiveness of cold and heat therapy in reducing pain in patients with DOMS.

Cold therapies include cold-water immersion (CWI), cold pack, ice massage, the novel modality of cryotherapy (CRYO) and phase change material (PCM). The immersion temperature of CWI is usually $\leq 15^{\circ} \mathrm{C}$; CRYO is a treatment involving very short exposures to extreme cold dry air to the whole patient or to a treatment area $(8,9)$. The intervention forms of CRYO include whole- and partial-body cryotherapy and air-pulsed cryotherapy, with the temperature of the cryotherapy chamber at $-30^{\circ} \mathrm{C},-80$ to $-110^{\circ} \mathrm{C}$, or $<-110^{\circ} \mathrm{C}$; the cold treatment temperature of $\mathrm{PCM}$ is $15^{\circ} \mathrm{C}(10)$. Cold treatment is thought to reduce swelling and cell metabolism, so that oedema, pain and injury are minimized (11).

Commonly used methods of heat therapy include hot/warm water immersion (HWI/WWI), hot pack, sauna and ultrasound. Heat treatment increases metabolism in tissues, promotes blood circulation and reduces pain (11). Another type of intervention is contrast water therapy (CWT), which is a combination therapy using cold and heat. The temperature range of cold therapy is generally less than or equal to $10^{\circ} \mathrm{C}$; and the temperature of the heat therapy $35-40^{\circ} \mathrm{C}$.

The effect of different methods of cold and heat therapy on pain in patients with DOMS is currently debated, and few studies have directly compared the effects of different cold and heat therapies; thus, the most effective cold and heat therapy for DOMS is unknown. Among them, there are many outcome variables for observing the effect of cold or heat therapy on DOMS, including subjective pain and objective indicators, such as creatine kinase (CK) and C-reactive protein (CRP), which are all important outcome observation indicators, and are independent of each other (12). However, in previous studies, reports of objective variables are inconsistent, but there are relatively numerous reports on the variable pain. Therefore, this study chose to conduct a network meta-analysis (NMA) on pain.
Using the same type of research object, an NMA can systematically compare several different types of intervention measures for a certain problem, and rank them according to the effect of a certain outcome index, to determine the best intervention scheme. NMA is used to evaluate the effectiveness of pain relief on different cold and heat methods in patients with DOMS, and to provide a basis for the clinical selection of appropriate cold and heat methods.

\section{METHODS}

The protocols followed were based on the preferred reporting item of the Preferred Reporting Items for Systematic Reviews and Meta Analyses (PRISMA) statement (13). This study also followed our protocol, registered in PROSPERO (ID CRD42020170632).

\section{Search strategy}

Searches were performed in the following databases: PubMed, CINAHL, the Cochrane Library, Web of Science and 4 Chinese databases (China National Knowledge Infrastructure (CNKI), VIP Database, Chinese Biomedical Database (CBM) and Wanfang Database), in order to conduct a comprehensive database retrieval, from the date of establishment of the database to 31 May 2021.

The following search keywords were used: "delayed onset muscle soreness", "DOMS", "muscle pain", "myalgia", "muscle soreness", "muscular pain", "muscular soreness", "sore muscle", "muscle tenderness", "muscle ache"; "cryotherapy", "ice", "cool", "cold", "cold therapy", "psychrotherapy", "frigotherapy", "cold temperature", "cold pack", "phase change material", "whole/partial-body cryotherapy", "ice massage"; "heat", "heating", "heat therapy", "heat treatment", "hot", "warm", "thermal therapy", "thermotherapy", "hyperthermia therapy", "induced hyperthermia", "hot temperature", "heat pack", "ultrasound", "spa", "sauna”, "shower", "steam", "steam bath"; "contrast water therapy", "hydrotherapy", "contrast therapy", "water immersion", "heating and cooling combination therapy", "whirlpool bath*". The search terms used in PubMed are shown as an example in Table I. An additional manual search of references included in the study was performed, in order to find other articles that may have been overlooked.

\section{Inclusion criteria}

The selection criteria used in this review were based on methodological and clinical factors, such as population, intervention, control, outcomes, and study design (PICOS) (13) criteria, as described below.

Participants. The patients included in the published articles were over 18 years of age, with DOMS after exercise. Inclusion of patients with DOMS was not restricted according to race, sex, nationality or profession.

Intervention. Studies needed to have participants receiving cold and heat therapy within $1 \mathrm{~h}$ after exercise (studies that repeated the intervention protocol on subsequent days were included). The treatment group intervention included cold, heat, or contrast water therapy (CWI, PCM, CWT, HWI/WWI, CRYO, cold pack, ice massage, hot pack, or ultrasound). 
Table I. Keywords used in the PubMed database

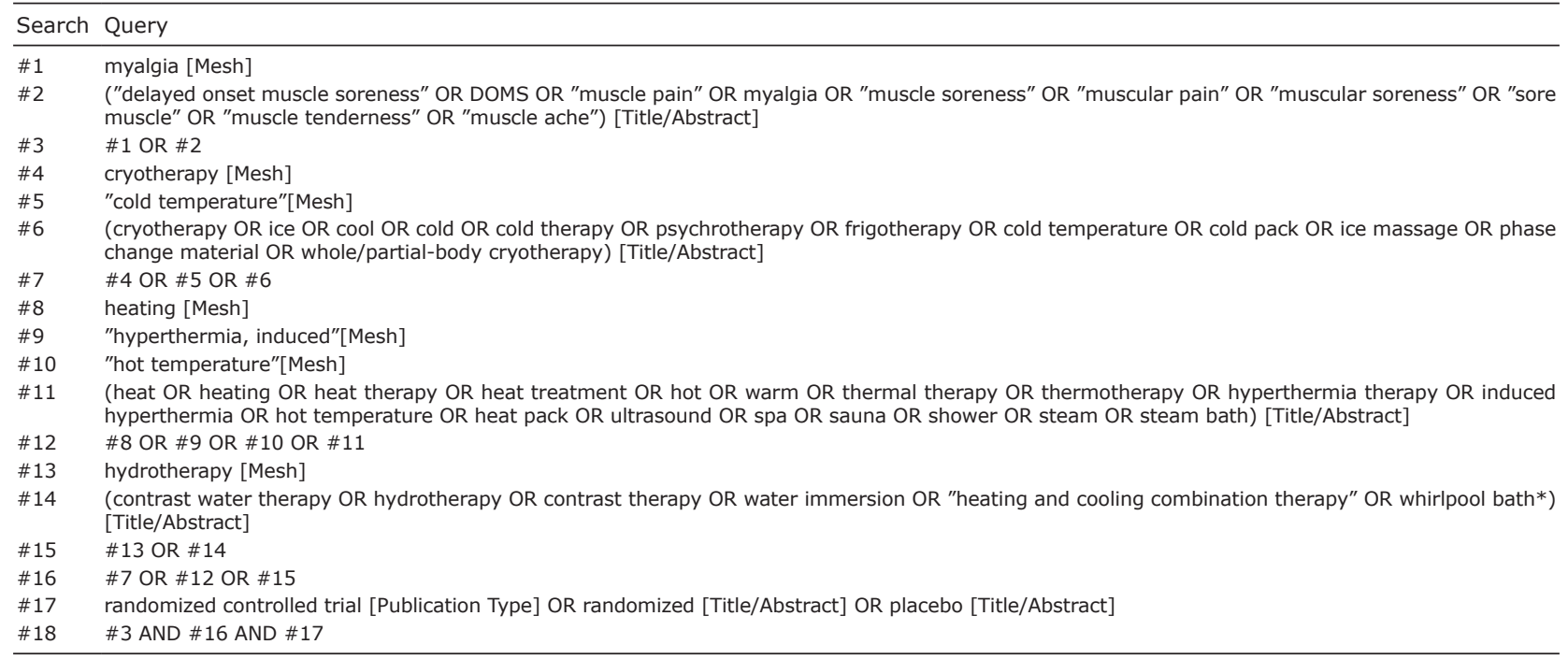

Comparisons. The control group interventions were passive recovery (PAS), including rest, no intervention or placebo.

Outcome. The outcome of DOMS was reported. Pain (muscle soreness) was measured on a visual analogue scale (VAS) pain score, Graphic Pain Rating Scale (GPRS), Likert scale, or modified Talag scale. Outcome variables were measured at baseline (pre-exercise) and post-exercise ( $24 \mathrm{~h}, 48 \mathrm{~h}$, and over $48 \mathrm{~h}$ follow-up time).

Study design. Randomized controlled trials (RCTs) were selected.

\section{Exclusion criteria}

Studies were excluded if: $(i)$ they were published in a language other than Chinese or English; (ii) re-published studies; (iii) there were insufficient data to report an effect size; (iv) they used multiple recovery modalities, including cold and heat treatment in conjunction with another recovery modality; or (v) participants had cardiovascular disease, hepatic disease, diabetes, obesity, etc.

\section{Study selection and data extraction}

The identified studies were selected by 2 authors independently. Titles and abstracts were scanned initially, and then the full articles were examined according to the inclusion criteria. In the case of disagreement, a third reviewer was consulted in order to reach a final consensus. Whenever clarification was needed, the paper's author was contacted for more information. Two reviewers independently extracted data from the included studies using standardized data extraction forms. For each study, data on the general characteristics of the study, research methods, participants, interventions, outcome measures, results, and other information were extracted.

\section{Risk of bias assessment}

Two reviewers independently assessed the risk of bias of included studies. According to the Cochrane Handbook for Systematic Reviews of Interventions (version 5.1.0) (14), this assessment tool covers 7 domains, including random sequence generation (selection bias), allocation concealment (selection bias), blinding of participants and personnel (performance bias), blinding of outcome assessment (detection bias), incomplete outcome data (attrition bias), selective reporting (reporting bias), and other bias. Risk of bias for each field was scored low, high, or unclear. Any disagreement between the 2 authors was resolved by consensus and discussion with a third reviewer. Publication bias was assessed using a funnel plot.

\section{Data synthesis and analysis}

All studies underwent multiple follow-up (pre- and post-exercise) observations for each outcome, and the mean difference of the pain score post-exercise $(24 \mathrm{~h}, 48 \mathrm{~h}$ and over $48 \mathrm{~h}$ followup time) from baseline (pre-exercise) change was calculated. Standardized mean difference (SMD) (different metrics are used across studies) and 95\% confidence interval (95\% CI) were used to assess the effects of different interventions on DOMS. Network meta-analysis compares multiple treatments simultaneously by combining direct and indirect evidence of the relative treatment effects. Stata software (version 16.0; StataCorp LLC, College Station, TX 77845, USA) was used to perform the network meta-analysis. The inconsistency factor (IFS) was used to estimate the inconsistency in each closed loop. If there was a closed loop in the network (for example, A-B-C), then each comparison in the loop (for example, A-B) may be the result of an indirect comparison (for example, A-C and C-B). Therefore, the results of direct and indirect comparisons may be different. The lower limit of $95 \%$ CI of the inconsistency factor (IF) is close to 0 , suggesting that the closed loop maintains consistency, with the consistency model selected for analysis. In the case of inconsistency, the inconsistency model was selected, and regression analysis or sensitivity analysis was used. Potential therapeutic benefits of interventions were ranked using surface area under the cumulative ranking curve (SUCRA) and MeanRank (15). The highest SUCRA score was $100 \%$. Higher SUCRA scores, smaller corresponding MeanRank values, and higher rankings indicate better efficacy. Publication bias was evaluated using a funnel plot. 


\section{RESULTS}

\section{Study identification and selection}

According to the retrieval strategy and data collection method, 1,807 reports were identified. After eliminating duplicate documents, a total of 1,389 articles were eligible. After reading the title and abstract, 150 studies were included in the full-text review. After further examining the remaining articles, another 91 studies were excluded for the reasons outlined in Fig. 1. A final total of 59 RCTs $(7-9,11$, 16-70) were included for review, comprising a total of 1,367 patients.

\section{Characteristics and risk of bias analysis of included studies}

The characteristics of the included studies are described in Table II. A summary of the risk of bias analyses for these studies is illustrated in Fig. 2.

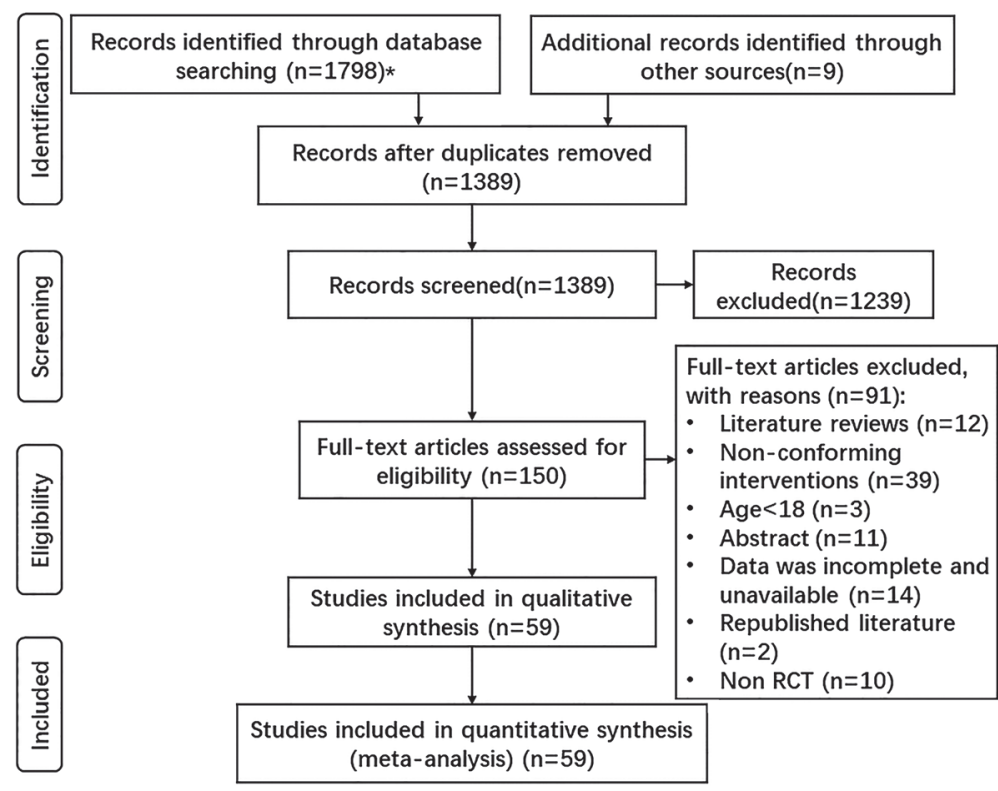

Fig. 1. Flowchart of literature search selection. Note: 59 studies that met the inclusion criteria were included in this network meta-analysis (NMA) and traditional meta-analysis. $\mathrm{n}$ : numbers. *PubMed $(n=197)$, Web of Science $(n=209)$, The Cochrane Library $(n=1,065)$, CINAHL $(n=207)$, Chinese Biomedical Database (CBM) $(n=40)$, China National Knowledge Infrastructure (CNKI) $(n=24)$, Wan Fang Data $(n=37)$ and VIP $(n=19)$.

\section{Publication bias and data consistency}

For the network meta-analysis, these funnel plots appeared slightly asymmetrical, which suggests possible publication bias or small sample size study effects (Fig. $3)$. According to the detection results of loop inconsistencies, the lower limit of $95 \%$ CI of the inconsistency factor (IF) was mostly close to 0 , which indicated that the results of most direct and indirect comparisons were consistent. Therefore, the consistency model was selected for analysis in this study.

\section{Network meta-analysis}

Network plots. Three comprehensive network graphs were built using Stata 16.0 (Fig. 4). The size of the circle represents the number of participants and the thickness of the edge represents the number of studies.
The interventions involved in the network graphs were CWI, PCM, HWI/WWI, CWT, CRYO, ice massage, cold pack, hot pack, ultrasound and PAS.

Comparative results of different interventions. See Table III, Table IV and Table V.

Interventions rank probability. The ranking probability of each treatment in terms of 3 follow-up times are shown in Fig. 5 and Table VI. Larger areas under the SUCRA curve and smaller MeanRank values indicate a better analgesic effect.

\section{DISCUSSION}

A total of 59 articles were included in this study. Through analysis of the subjective scores of patients with DOMS after exercise, the analgesic effects of 9 cold
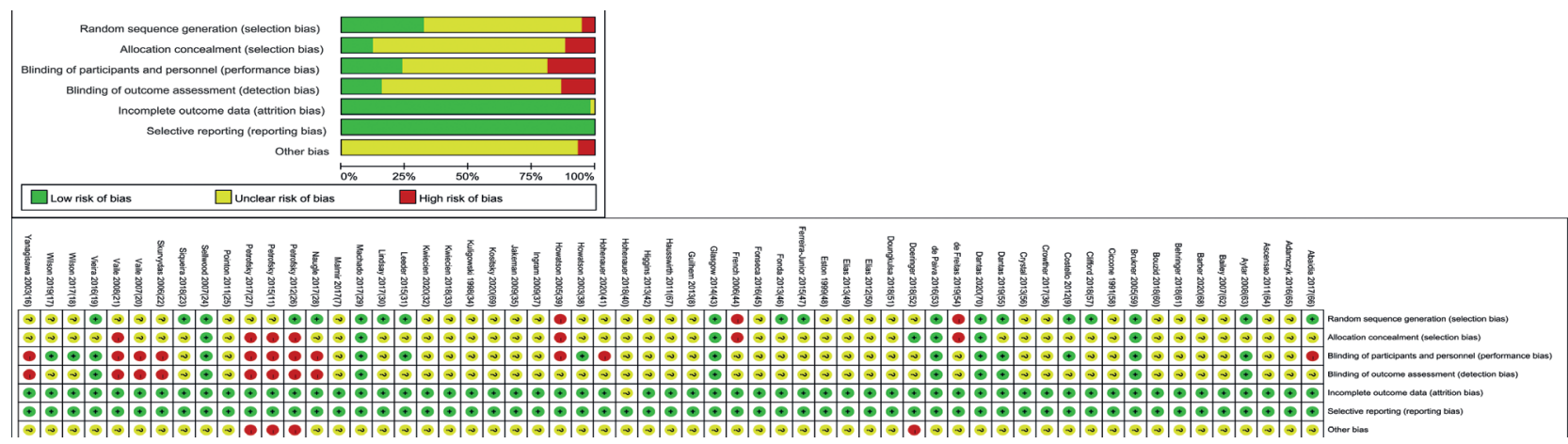

Fig. 2. Risk of bias graph (top), and risk of bias summary (bottom). 
Table II. Characteristics of the included studies

\begin{tabular}{|c|c|c|c|c|c|c|c|c|}
\hline \multirow[b]{2}{*}{ Reference } & \multirow[b]{2}{*}{ Country } & \multirow{2}{*}{$\begin{array}{l}\text { Number of cases } \\
(T / C)\end{array}$} & \multirow[b]{2}{*}{ Sex (male/female) } & \multirow[b]{2}{*}{ Profession } & \multicolumn{2}{|c|}{ Mean age, years } & \multicolumn{2}{|c|}{ Interventions* } \\
\hline & & & & & $\mathrm{T}$ & $\mathrm{C}$ & $T$ & $\mathrm{C}$ \\
\hline Abaidia et al. 2017 (66) & FR/UK/AUS & $10 / 10$ & $10 / 0$ & Athletes & $23.40 \pm 4.00$ & & CWI & WBC \\
\hline Adamczyk et al. 2016 (65) & PL/AUS & $12 / 12$ & $36 / 0$ & Non-athletes & $22.50 \pm 0.90$ & $22.10 \pm 0.70$ & Ice massage & PAS \\
\hline & & $12 / 12$ & & Non-athletes & $22.50 \pm 0.80$ & & CWI & \\
\hline Ascensao et al. 2011 (64) & PT & $10 / 10$ & $20 / 0$ & Athletes & $18.10 \pm 1.80$ & $18.30 \pm 0.80$ & CWI & HWI \\
\hline Aytar et al. 2008 (63) & TR & $30 / 30$ & $0 / 90$ & Non-athletes & $22.00 \pm 2.08$ & $21.60 \pm 2.14$ & Ultrasound & PAS \\
\hline Bailey et al. 2007 (62) & UK & $10 / 10$ & $20 / 0$ & Non-athletes & $23.60 \pm 4.10$ & $21.70 \pm 2.00$ & CWI & PAS \\
\hline Barber et al. 2020 (68) & UK & $8 / 8$ & $16 / 0$ & Athletes & $20.00 \pm 1.20$ & & CWI & PAS \\
\hline Behringer et al. 2018 (61) & $\mathrm{DE}$ & $11 / 8$ & $21 / 9$ & Athletes & $26.10 \pm 3.00$ & $24.00 \pm 1.70$ & Cold pack & PAS \\
\hline Bouzid et al. $2018(60)$ & TN & $8 / 8$ & $8 / 0$ & Athletes & $19.63 \pm 0.74$ & & CWI & WWI \\
\hline Ciccone et al. 1991 (58) & USA & $10 / 10$ & $0 / 40$ & Non-athletes & - & - & ultrasound & PAS \\
\hline Clifford et al. 2018 (57) & UK/USA & $11 / 11$ & $11 / 0$ & Athletes & $19.00 \pm 1.00$ & & PCM & PAS \\
\hline Costello et al. 2012 (9) & $\mathrm{IE}$ & $9 / 9$ & - & Non-athletes & $21.20 \pm 2.10$ & & CRYO & PAS \\
\hline Crystal et al. 2013 (56) & USA & $10 / 10$ & $20 / 0$ & Non-athletes & $20.90 \pm 0.90$ & $21.50 \pm 3.20$ & CWI & PAS \\
\hline Crowther et al. 2017 (36) & AUS & $29 / 29$ & $29 / 0$ & Non-athletes & $27.00 \pm 6.00$ & & CWI & PAS \\
\hline & & $29 / 29$ & & Non-athletes & & & CWT & \\
\hline Dantas et al. 2019 (55) & $B R$ & $10 / 10$ & $20 / 0$ & Non-athletes & $30.28 \pm 6.10$ & $33.00 \pm 4.84$ & CWI & PAS \\
\hline de Paiva et al. 2016 (53) & $\mathrm{BR} / \mathrm{USA}$ & $10 / 10$ & $50 / 0$ & Non-athletes & - & - & Cold pack & PAS \\
\hline Doeringer et al. 2018 (52) & USA & $12 / 10$ & $7 / 15$ & Athletes & - & - & CWI & PAS \\
\hline Doungkulsa et al. 2018 (51) & TH/UK & $16 / 16$ & $32 / 0$ & Non-athletes & $21.31 \pm 1.03$ & & CRYO & PAS \\
\hline Dantas et al. $2020(70)$ & $\mathrm{BR}$ & $10 / 10$ & $20 / 0$ & Non-athletes & $30.00 \pm 6.09$ & $31.00 \pm 4.80$ & CWI & PAS \\
\hline Elias et al. 2013 (49) & AUS & $8 / 8$ & $24 / 0$ & Athletes & $19.90 \pm 2.80$ & & CWI & PAS \\
\hline & & $8 / 8$ & & Athletes & & & CWT & \\
\hline Elias et al. 2012 (50) & AUS & $14 / 14$ & $14 / 0$ & Athletes & $20.90 \pm 3.30$ & & CWI & PAS \\
\hline & & $14 / 14$ & & Athletes & & & CWT & \\
\hline Eston et al. 1999 (48) & UK & $8 / 7$ & $0 / 15$ & Non-athletes & $22.00 \pm 2.00$ & & CWI & PAS \\
\hline Ferreira-Junior et al. 2015 (47) & BR/USA & $13 / 13$ & $26 / 0$ & Non-athletes & $20.20 \pm 2.70$ & $20.30 \pm 2.20$ & CRYO & PAS \\
\hline Fonda et al. 2013 (46) & SI & $11 / 11$ & $11 / 0$ & Non-athletes & $26.90 \pm 3.80$ & & CRYO & PAS \\
\hline Fonseca et al. 2016 (45) & $\mathrm{BR}$ & $4 / 4$ & $8 / 0$ & Athletes & $24.00 \pm 3.60$ & & CWI & PAS \\
\hline French et al. 2006 (44) & UK & $10 / 6$ & $26 / 0$ & Athletes & $23.90 \pm 4.90$ & $21.50 \pm 2.00$ & CWT & PAS \\
\hline Glasgow et al. 2014 (43) & UK & $20 / 10$ & $32 / 18$ & Non-athletes & $18-35$ & & CWI & PAS \\
\hline Guilhem et al. 2013 (8) & FR/AUS & $12 / 12$ & $24 / 0$ & Non-athletes & $25.20 \pm 1.10$ & $23.90 \pm 1.40$ & CRYO & PAS \\
\hline Hausswirth et al. 2011 (67) & FR & $9 / 9$ & - & Athletes & $31.80 \pm 6.50$ & & CRYO & PAS \\
\hline & & $9 / 9$ & - & Athletes & & & Ultrasound & \\
\hline Higgins et al. 2013 (42) & AUS & $8 / 8$ & $24 / 0$ & Athletes & $19.50 \pm 0.80$ & & CWI & PAS \\
\hline & & $8 / 8$ & & Athletes & & & CWT & \\
\hline Howatson et al. 2005 (39) & UK & $12 / 12$ & $12 / 0$ & Non-athletes & $24.80 \pm 5.30$ & & Ice massage & PAS \\
\hline Howatson et al. 2003 (38) & UK & $9 / 9$ & $9 / 0$ & Athletes & $23.30 \pm 3.00$ & & Ice massage & PAS \\
\hline Ingram et al. 2009 (37) & AUS & $11 / 11$ & $11 / 0$ & Athletes & $27.50 \pm 6.00$ & & CWT & PAS \\
\hline & & $11 / 11$ & & Athletes & & & CWI & \\
\hline Jakeman et al. 2009 (35) & UK & $9 / 9$ & $0 / 18$ & Athletes & $19.90+0.97$ & & CWI & PAS \\
\hline Kositsky et al. 2020 (69) & FI & $5 / 5$ & $10 / 0$ & Athletes & $19.40 \pm 0.90$ & $18.4 \pm 0.50$ & CWI & PAS \\
\hline Kuligowski et al. 1998 (34) & USA & $14 / 14$ & $28 / 28$ & Non-athletes & - & - & WWI & PAS \\
\hline & & $14 / 14$ & & Non-athletes & & & CWI & \\
\hline & & $14 / 14$ & & Non-athletes & & & CWT & \\
\hline Kwiecien et al. 2018 (33) & USA/UK/ZA & $4 / 8$ & $6 / 2$ & Non-athletes & $37.00 \pm 12.00$ & & PCM & PAS \\
\hline Kwiecien et al. 2020 (32) & USA/UK/ZA & $13 / 13$ & $26 / 0$ & Athletes & $25.00 \pm 6.00$ & & PCM & PAS \\
\hline Leeder et al. 2015 (31) & UK/ZA & $8 / 8$ & $24 / 0$ & Athletes & $22.00 \pm 3.00$ & $22.00 \pm 3.00$ & CWI & PAS \\
\hline Lindsay et al. 2017 (30) & USA/NZ & $7 / 8$ & $15 / 0$ & Athletes & $28.30 \pm 5.70$ & & CWI & PAS \\
\hline Machado et al. 2017 (29) & $\mathrm{BR}$ & $40 / 20$ & $60 / 0$ & - & $21.00 \pm 2.25$ & $20.40 \pm 1.80$ & CWI & PAS \\
\hline Malmir et al. 2017 (7) & IR & $16 / 10$ & - & $\begin{array}{l}\text { Amateur } \\
\text { athletes }\end{array}$ & $26.00 \pm 3.00$ & $26.00 \pm 3.00$ & Cold pack & PAS \\
\hline Naugle et al. 2017 (28) & USA & $12 / 13$ & $4 / 45$ & Non-athletes & $20.00 \pm 1.90$ & & Cold pack & PAS \\
\hline Petrofsky et al. 2017 (27) & USA/KOR & $20 / 20$ & - & Non-athletes & $26.00 \pm 2.60$ & $25.30 \pm 3.00$ & Hot pack & PAS \\
\hline Petrofsky et al. 2015 (11) & USA & $20 / 20$ & - & Non-athletes & $25.50 \pm 2.70$ & $25.30 \pm 30$ & Cold pack & PAS \\
\hline & & $20 / 20$ & - & Non-athletes & $26.10 \pm 2.60$ & & Hot pack & \\
\hline Petrofsky et al. 2012 (26) & USA & $5 / 5$ & $8 / 12$ & Athletes & $25.80 \pm 3.10$ & $26.50 \pm 13.30$ & Hot pack & PAS \\
\hline Pointon et al. 2011 (25) & AUS & $10 / 10$ & $10 / 0$ & Athletes & $21.00 \pm 1.60$ & & Cold pack & PAS \\
\hline Sellwood et al. 2007 (24) & AUS & $20 / 20$ & $11 / 29$ & Non-athletes & $21.40 \pm 4.30$ & $21.00 \pm 3.10$ & CWI & WWI \\
\hline Siqueira et al. 2018 (23) & $\mathrm{BR}$ & $15 / 15$ & $30 / 0$ & Non-athletes & $20.50 \pm 1.40$ & $19.90 \pm 1.40$ & CWI & PAS \\
\hline Skurvydas et al. 2006 (22) & $\mathrm{LT}$ & $20 / 20$ & $20 / 0$ & Non-athletes & $20.40 \pm 1.70$ & & CWI & PAS \\
\hline Vaile 2008 et al. (21) & AUS/NZ & $12 / 38$ & $38 / 0$ & Non-athletes & - & - & CWI & PAS \\
\hline & & $11 / 38$ & & Non-athletes & - & - & HWI & \\
\hline & & $15 / 38$ & & Non-athletes & - & - & CWT & \\
\hline Vaile 2007 et al. (20) & AUS/NZ/UK & $13 / 13$ & $4 / 9$ & Non-athletes & $26.20 \pm 5.80$ & $26.20 \pm 5.80$ & CWT & PAS \\
\hline Vieira 2016 et al. (19) & BR/AUS & $28 / 14$ & $42 / 0$ & Non-athletes & $21.15 \pm 2.50$ & $20.20 \pm 1.70$ & CWI & PAS \\
\hline Wilson 2017 et al. (18) & UK & $11 / 10$ & $31 / 0$ & Non-athletes & $41.30 \pm 7.60$ & $40.60 \pm 7.20$ & CWI & PAS \\
\hline & & $10 / 10$ & & Non-athletes & $37.70 \pm 8.90$ & & CRYO & \\
\hline Wilson 2019 et al. (17) & UK & $8 / 8$ & $24 / 0$ & Non-athletes & $21.88 \pm 3.40$ & $25.88 \pm 5.19$ & CWI & PAS \\
\hline & & $8 / 8$ & & Non-athletes & $26.50 \pm 8.40$ & & CRYO & \\
\hline Yanagisawa 2003 et al. (16) & $\mathrm{JP}$ & $9 / 10$ & $28 / 0$ & Non-athletes & $23.80 \pm 1.80$ & & CWI & PAS \\
\hline
\end{tabular}

AUS: Australia; BE: Belgium; BR: Brazil; CA: Canada; CH: Switzerland; DE: Germany; FI: Finland; FR: France; IE: Ireland; IR: Iran; JP: Japan; KOR: South Korea; LT:
Lithuania; LU: Luxembourg; NZ: New Zealand; PL: Poland; PT: Portugal; SI: Slovakia; TN: Tunisia; TR: Turkey; TH: Thailand; ZA: South Africa; -: not mentioned. *All studies applied the intervention measures within $1 \mathrm{~h}$ after exercise. PAS: (Passive recovery: rest, no intervention or placebo). CWI (cold water immersion; CRYO (the novel modality of cryotherapy; PCM (phase change material; CWT (contrast water therapy); and HWI/WWI (hot/warm water immersion). 

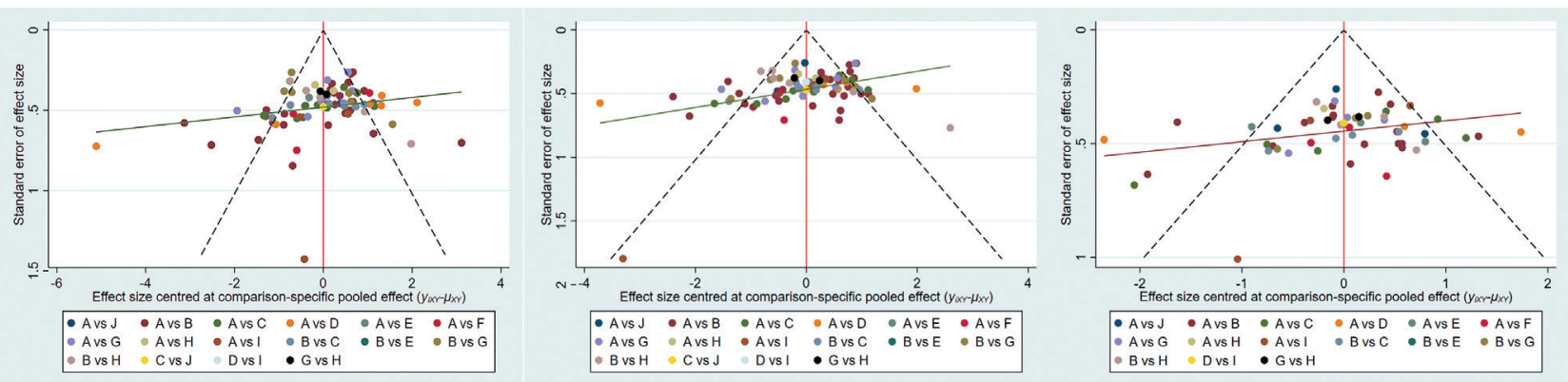

Fig. 3. Funnel plot of the efficacy of 7 interventions of cold and heat therapies and one passive recovery (control group). Left: $24 \mathrm{~h}$; middle: 48 h; right: $>48 \mathrm{~h}$. A: passive recovery (PAS); B: cold water immersion (CWI); C: the novel modality of cryotherapy (CRYO); D: cold pack, E: ice massage, F: phase change material (PCM); G: contrast water therapy (CWT); H: hot/warm water immersion (HWI/WWI); I: hot pack, J: ultrasound.

and heat therapy interventions and 1 passive recovery were compared. A total of 10 interventions were included in the network meta-analysis: CWI, CRYO, PCM, CWT, HWI/WWI, cold pack, hot pack, ice massage, ultrasound and PAS (7-9, 11, 16-70). The use of hot pack within $1 \mathrm{~h}$ after exercise resulted in the best pain relief within $48 \mathrm{~h}$ post-exercise. Use of CRYO within $1 \mathrm{~h}$ after exercise has a significant effect on pain relief over $48 \mathrm{~h}$ post-exercise. At the $24 \mathrm{~h}$ follow-up after exercise, the 10 interventions were ranked as follows: hot pack, CWT, CRYO, PCM, cold pack, CWI, ice massage, ultrasound, HWI/WWI, PAS. At $48 \mathrm{~h}$ postexercise, the ranking was: hot pack, CRYO, PCM, CWT, ultrasound, CWI, HWI/WWI, cold pack, ice massage, PAS. At over $48 \mathrm{~h}$, the ranking of analgesic effect was: CRYO, PCM, CWT, hot pack, ice massage, CWI, cold pack, HWI/WWI, ultrasound, PAS.

Hot pack is one of the oldest interventions in cold and heat therapies. Compared with other interventions, the technology is safer and more mature. Hot pack can resist the loss of heat after exercise, keep tissue warm, increase blood flow speed and metabolism, and speed up clearance of inflammatory factors, thus reducing pain in patients (27). In the network meta-analysis, the results show that the effect of hot pack is ranked the first within $48 \mathrm{~h}$. Several previous studies $(4,26$, 71) have also found that thermal therapy is effective in reducing acute, non-specific low back pain and pain in patients with DOMS. The reason may be the increasing skin temperature $24 \mathrm{~h}$ after exercise in all subjects, probably due to higher blood flow in muscle, inflammation, and repair of tissue damage caused by the exercise. Therefore, timely heat therapy after exercise can reduce the loss of muscle tissue temperature, maintain a constant temperature in the tissue, promote blood circulation to the affected area, increase the supply of nutrients and oxygen to the injured area, accelerate metabolism, and reduce peripheral nerve excitability, thereby alleviating pain. However, studies have shown that heat pack only changes the temperature of the subcutaneous tissue to a depth of 1-2 cm below the skin surface, suggesting that any temporary effects related to pain relief after intervention may soon wear off. Therefore, hot pack may only have a better effect on pain within $48 \mathrm{~h}$ after exercise. However, there were few studies of the application of hot pack for DOMS, and the difference between the temperature and form of hot pack may cause inconsistency in the study, affecting the stability of the results. Thus, the results should be treated with caution. More relevant clinical RCTs of hot packs are required in the future.

The analgesic effect of CRYO (the novel modality of cryotherapy) was gradually superior to other intervention measures, with an increase in rest time
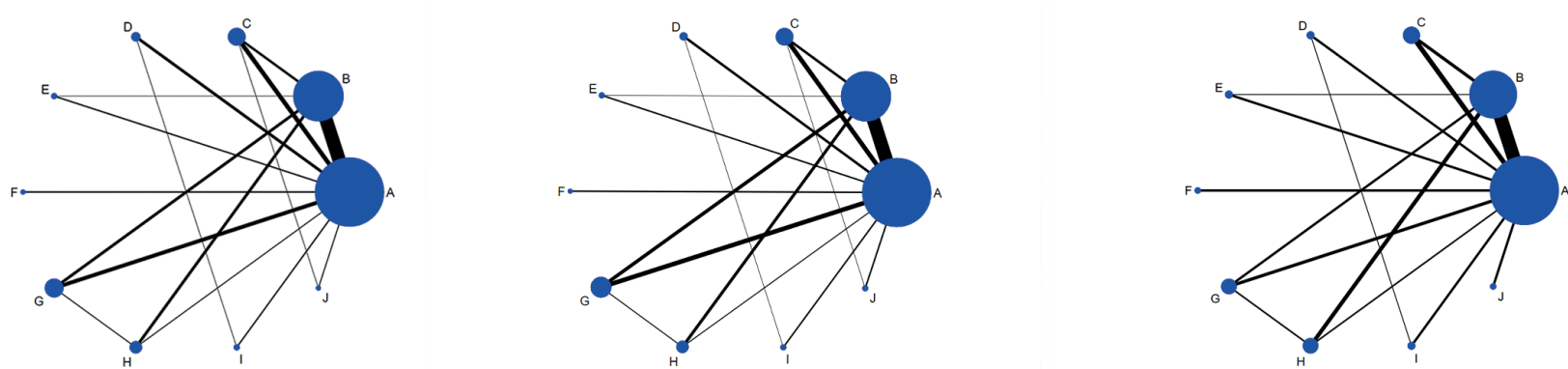

Fig. 4. Network plot of 10 treatment methods. Left: within $24 \mathrm{~h}$ follow-up time; middle: within $48 \mathrm{~h}$ follow-up time; right: $>48 \mathrm{~h}$ follow-up time. A: passive recovery (PAS); B: cold water immersion (CWI); C: the novel modality of cryotherapy (CRYO); D: cold pack, E: ice massage, F: phase change material (PCM); G: contrast water therapy (CWT); H: hot/warm water immersion (HWI/WWI); I: hot pack, J: ultrasound. 
Table III. Follow-up time: 24 h-network meta-analysis of the different interventions to alleviate the pain of delayed-onset muscle soreness (DOMS) (standardized mean difference (SMD) (95\% confidence interval; $95 \% \mathrm{CI})$ ).

\begin{tabular}{|c|c|c|c|c|c|c|c|c|c|}
\hline \multicolumn{10}{|l|}{ Ultrasound } \\
\hline \begin{tabular}{|l|}
4.41 \\
$(1.70,7.12)$ \\
\end{tabular} & Hot pack & & & & & & & & \\
\hline $\begin{array}{l}-0.28 \\
(-2.55 \text { to } 1.98)\end{array}$ & $\begin{array}{l}-4.69 \\
(-7.02 \text { to }-2.37)\end{array}$ & HWI/WWI & & & & & & & \\
\hline $\begin{array}{l}1.03 \\
(-1.07 \text { to } 3.13)\end{array}$ & $\begin{array}{l}-3.38 \\
(-5.54 \text { to }-1.22)\end{array}$ & $\begin{array}{l}1.31 \\
(-0.25 \text { to } 2.88)\end{array}$ & CWT & & & & & & \\
\hline $\begin{array}{l}0.91 \\
(-1.28 \text { to } 3.11)\end{array}$ & $\begin{array}{l}-3.50 \\
(-5.75 \text { to }-1.24)\end{array}$ & $\begin{array}{l}1.20 \\
(-0.50 \text { to } 2.89)\end{array}$ & $\begin{array}{l}-0.12 \\
(-1.58 \text { to } 1.34)\end{array}$ & PCM & & & & & \\
\hline $\begin{array}{l}0.51 \\
(-1.55 \text { to } 2.57)\end{array}$ & $\begin{array}{l}-3.90 \\
(-6.02 \text { to }-1.77)\end{array}$ & $\begin{array}{l}0.80 \\
(-0.72 \text { to } 2.31)\end{array}$ & $\begin{array}{l}-0.52 \\
(-1.77 \text { to } 0.73)\end{array}$ & $\begin{array}{l}-0.40 \\
(-1.81 \text { to } 1.01)\end{array}$ & $\begin{array}{l}0.34 \\
(-1.67 \text { to } 2.35)\end{array}$ & Cold pack & & & \\
\hline $\begin{array}{l}0.97 \\
(-1.22 \text { to } 3.16)\end{array}$ & $\begin{array}{l}-3.44 \\
(-5.69 \text { to }-1.19)\end{array}$ & $\begin{array}{l}1.25 \\
(-0.43 \text { to } 2.93)\end{array}$ & $\begin{array}{l}-0.06 \\
(-1.51 \text { to } 1.39)\end{array}$ & $\begin{array}{l}0.06 \\
(-1.53 \text { to } 1.64)\end{array}$ & $\begin{array}{l}0.80 \\
(-1.34 \text { to } 2.94)\end{array}$ & $\begin{array}{l}0.46 \\
(-0.94 \text { to } 1.85)\end{array}$ & CRYO & & \\
\hline $\begin{array}{l}0.25 \\
(-1.68 \text { to } 2.19)\end{array}$ & $\begin{array}{l}-4.16 \\
(-6.16 \text { to }-2.15)\end{array}$ & $\begin{array}{l}0.54 \\
(-0.80 \text { to } 1.88)\end{array}$ & $\begin{array}{l}-0.78 \\
(-1.81 \text { to } 0.25)\end{array}$ & $\begin{array}{l}-0.66 \\
(-1.88 \text { to } 0.56)\end{array}$ & $\begin{array}{l}0.08 \\
(-1.80 \text { to } 1.96)\end{array}$ & $\begin{array}{l}-0.26 \\
(-1.22 \text { to } 0.70)\end{array}$ & $\begin{array}{l}-0.72 \\
(-1.92 \text { to } 0.49)\end{array}$ & CWI & \\
\hline $\begin{array}{l}-1.19(-2.31 \text { to } \\
-0.08)\end{array}$ & $\begin{array}{l}1.01 \\
(-0.78 \text { to } 2.80)\end{array}$ & $\begin{array}{l}0.43 \\
(-1.76 \text { to } 2.63)\end{array}$ & $\begin{array}{l}0.51 \\
(-0.88 \text { to } 1.90)\end{array}$ & $\begin{array}{l}-0.48 \\
(-0.93 \text { to }-0.02)\end{array}$ & $\begin{array}{l}0.36 \\
(-0.98 \text { to } 1.70)\end{array}$ & $\begin{array}{l}-1.52 \\
(-2.59 \text { to }-0.45)\end{array}$ & $\begin{array}{l}0.18 \\
(-1.70 \text { to } 2.06)\end{array}$ & $\begin{array}{l}-0.11 \\
(-1.30 \text { to } 1.09)\end{array}$ & PAS \\
\hline
\end{tabular}

Table IV. Follow-up time: $48 \mathrm{~h}$ network meta-analysis of the different interventionstoalleviate the pain of DOMS (SMD (95\%CI)).

\begin{tabular}{|c|c|c|c|c|c|c|c|c|c|}
\hline \multicolumn{10}{|l|}{ Ultrasound } \\
\hline $\begin{array}{l}1.68 \\
(-0.68 \text { to } 4.05)\end{array}$ & Hot pack & & & & & & & & \\
\hline $\begin{array}{l}-0.17 \\
(-2.23 \text { to } 1.89)\end{array}$ & $\begin{array}{l}-1.85 \\
(-3.83 \text { to } 0.13)\end{array}$ & HWI/WWI & & & & & & & \\
\hline $\begin{array}{l}0.40 \\
(-1.48 \text { to } 2.27)\end{array}$ & $\begin{array}{l}-1.29 \\
(-3.08 \text { to } 0.51)\end{array}$ & $\begin{array}{l}0.57 \\
(-0.79 \text { to } 1.93)\end{array}$ & CWT & & & & & & \\
\hline $\begin{array}{l}0.47 \\
(-1.53 \text { to } 2.47)\end{array}$ & $\begin{array}{l}-1.21 \\
(-3.13 \text { to } 0.71)\end{array}$ & $\begin{array}{l}0.64 \\
(-0.88 \text { to } 2.17)\end{array}$ & $\begin{array}{l}0.08 \\
(-1.20 \text { to } 1.35)\end{array}$ & PCM & & & & & \\
\hline $\begin{array}{l}-0.32 \\
(-2.70 \text { to } 2.06)\end{array}$ & $\begin{array}{l}-2.01 \\
(-4.32 \text { to } 0.31)\end{array}$ & $\begin{array}{l}-0.15 \\
(-2.15 \text { to } 1.85)\end{array}$ & $\begin{array}{l}-0.72 \\
(-2.53 \text { to } 1.09)\end{array}$ & $\begin{array}{l}-0.80 \\
(-2.73 \text { to } 1.14)\end{array}$ & Ice massage & & & & \\
\hline $\begin{array}{l}-0.19 \\
(-2.10 \text { to } 1.72)\end{array}$ & $\begin{array}{l}-1.87 \\
(-3.70 \text { to }-0.04)\end{array}$ & $\begin{array}{l}-0.02 \\
(-1.43 \text { to } 1.39)\end{array}$ & $\begin{array}{l}-0.59 \\
(-1.72 \text { to } 0.54)\end{array}$ & $\begin{array}{l}-0.66 \\
(-1.99 \text { to } 0.66)\end{array}$ & $\begin{array}{l}0.13 \\
(-1.72 \text { to } 1.98)\end{array}$ & Cold pack & & & \\
\hline $\begin{array}{l}0.77 \\
(-1.23 \text { to } 2.77)\end{array}$ & $\begin{array}{l}-0.91 \\
(-2.83 \text { to } 1.01)\end{array}$ & $\begin{array}{l}0.95 \\
(-0.58 \text { to } 2.47)\end{array}$ & $\begin{array}{l}0.38 \\
(-0.90 \text { to } 1.65)\end{array}$ & $\begin{array}{l}0.30 \\
(-1.15 \text { to } 1.75)\end{array}$ & $\begin{array}{l}1.10 \\
(-0.84 \text { to } 3.04)\end{array}$ & $\begin{array}{l}0.96 \\
(-0.36 \text { to } 2.29)\end{array}$ & CRYO & & \\
\hline $\begin{array}{l}-0.01 \\
(-1.78 \text { to } 1.76)\end{array}$ & \begin{tabular}{|l|}
-1.69 \\
$(-3.37$ to -0.01$)$
\end{tabular} & $\begin{array}{l}0.16 \\
(-1.05 \text { to } 1.38)\end{array}$ & $\begin{array}{l}-0.40 \\
(-1.27 \text { to } 0.46)\end{array}$ & $\begin{array}{l}-0.48 \\
(-1.59 \text { to } 0.63)\end{array}$ & $\begin{array}{l}0.31 \\
(-1.39 \text { to } 2.02)\end{array}$ & $\begin{array}{l}0.18 \\
(-0.76 \text { to } 1.13)\end{array}$ & $\begin{array}{l}-0.78 \\
(-1.89 \text { to } 0.33)\end{array}$ & CWI & \\
\hline $\begin{array}{l}-1.40 \\
(-2.42 \text { to }-0.37)\end{array}$ & $\begin{array}{l}1.02 \\
(-0.62 \text { to } 2.66)\end{array}$ & $\begin{array}{l}0.58 \\
(-1.43 \text { to } 2.58)\end{array}$ & $\begin{array}{l}0.66 \\
(-0.61 \text { to } 1.94)\end{array}$ & $\begin{array}{l}-0.61 \\
(-1.04 \text { to }-0.18)\end{array}$ & $\begin{array}{l}0.43 \\
(-0.78 \text { to } 1.64)\end{array}$ & $\begin{array}{l}-0.70 \\
(-1.59 \text { to } 0.19)\end{array}$ & $\begin{array}{l}-0.02 \\
(-1.73 \text { to } 1.69)\end{array}$ & $\begin{array}{l}-0.29 \\
(-1.40 \text { to } 0.81)\end{array}$ & PAS \\
\hline
\end{tabular}

Table V. Follow-up time: over $48 \mathrm{~h}$ network meta-analysis of the different interventionstoalleviate the pain of DOMS (SMD (95\%CI)

\begin{tabular}{|c|c|c|c|c|c|c|c|c|c|}
\hline Ultrasound & & & & & & & & & \\
\hline $\begin{array}{l}0.83 \\
(-0.59 \text { to } 2.25)\end{array}$ & Hot pack & & & & & & & & \\
\hline $\begin{array}{l}0.11 \\
(-1.04 \text { to } 1.26)\end{array}$ & \begin{tabular}{|l}
-0.72 \\
$(-2.22$ to 0.79$)$
\end{tabular} & HWI/WWI & & & & & & & \\
\hline $\begin{array}{l}1.04 \\
(-0.62 \text { to } 2.69)\end{array}$ & $\begin{array}{l}0.21 \\
(-1.71 \text { to } 2.12)\end{array}$ & $\begin{array}{l}0.93 \\
(-0.80 \text { to } 2.65)\end{array}$ & CWT & & & & & & \\
\hline $\begin{array}{l}0.93 \\
(-0.18 \text { to } 2.04)\end{array}$ & $\begin{array}{l}0.10 \\
(-1.37 \text { to } 1.58)\end{array}$ & $\begin{array}{l}0.82 \\
(-0.39 \text { to } 2.03)\end{array}$ & $\begin{array}{l}-0.11 \\
(-1.80 \text { to } 1.59)\end{array}$ & PCM & & & & & \\
\hline $\begin{array}{l}0.07 \\
(-1.12 \text { to } 1.27)\end{array}$ & $\begin{array}{l}-0.75 \\
(-2.30 \text { to } 0.79) \\
\end{array}$ & $\begin{array}{l}-0.03 \\
(-1.33 \text { to } 1.26) \\
\end{array}$ & $\begin{array}{l}-0.96 \\
(-2.72 \text { to } 0.79) \\
\end{array}$ & $\begin{array}{l}-0.85 \\
(-2.11 \text { to } 0.41) \\
\end{array}$ & $\begin{array}{l}-0.63 \\
(-2.25 \text { to } 1.00) \\
\end{array}$ & Cold pack & & & \\
\hline $\begin{array}{l}1.55 \\
(0.23 \text { to } 2.88)\end{array}$ & $\begin{array}{l}0.72 \\
(-0.92 \text { to } 2.37)\end{array}$ & $\begin{array}{l}1.44 \\
(0.03 \text { to } 2.86) \\
\end{array}$ & $\begin{array}{l}0.52 \\
(-1.33 \text { to } 2.36)\end{array}$ & $\begin{array}{l}0.62 \\
(-0.76 \text { to } 2.01)\end{array}$ & $\begin{array}{l}0.85 \\
(-0.87 \text { to } 2.58)\end{array}$ & $\begin{array}{l}1.48 \\
(0.02 \text { to } 2.93)\end{array}$ & CRYO & & \\
\hline $\begin{array}{l}0.19 \\
(-0.65 \text { to } 1.03)\end{array}$ & $\begin{array}{l}-0.64 \\
(-1.92 \text { to } 0.64)\end{array}$ & $\begin{array}{l}0.08 \\
(-0.89 \text { to } 1.05)\end{array}$ & $\begin{array}{l}-0.85 \\
(-2.38 \text { to } 0.69)\end{array}$ & $\begin{array}{l}-0.74 \\
(-1.66 \text { to } 0.18)\end{array}$ & $\begin{array}{l}-0.51 \\
(-1.90 \text { to } 0.87)\end{array}$ & $\begin{array}{l}0.11 \\
(-0.91 \text { to } 1.14)\end{array}$ & $\begin{array}{l}-1.36 \\
(-2.54 \text { to }-0.19)\end{array}$ & CWI & \\
\hline $\begin{array}{l}-3.50 \\
(-5.19 \text { to }-1.81)\end{array}$ & $\begin{array}{l}-1.81 \\
(-2.92 \text { to }-0.71)\end{array}$ & $\begin{array}{l}1.29 \\
(-0.23 \text { to } 2.81)\end{array}$ & $\begin{array}{l}1.42 \\
(0.13 \text { to } 2.71)\end{array}$ & $\begin{array}{l}-0.45 \\
(-0.85 \text { to }-0.05)\end{array}$ & $\begin{array}{l}-0.21 \\
(-1.17 \text { to } 0.76)\end{array}$ & $\begin{array}{l}0.12 \\
(-1.37 \text { to } 1.60)\end{array}$ & $\begin{array}{l}-0.09 \\
(-1.46 \text { to } 1.28)\end{array}$ & $\begin{array}{l}-0.60 \\
(-1.74 \text { to } 0.53)\end{array}$ & PAS \\
\hline
\end{tabular}

PAS: passive recovery; CWI: cold water immersion; CRYO: the novel modality of cryotherapy; PCM: phase change material; CWT: contrast water therapy; HWI/ WWI: hot/warm water immersion). Statistically significant findings are in bold: when the $95 \%$ CI did not contain " 0 ", pairwise comparison of interventions is statistically significant, i.e., $p$-value $<0.05$; when the $95 \%$ CI contained 0 , pairwise comparison of interventions is not statistically significant, i.e., $p$-value $\geq 0.05$ ). $\mathrm{SMD}<0$ indicates that the intervention in the column is more effective than the intervention in the line in reducing the level of pain; $\mathrm{SMD}>0$ indicates that the intervention in the line is more effective than the intervention in the column in reducing the level of pain. 

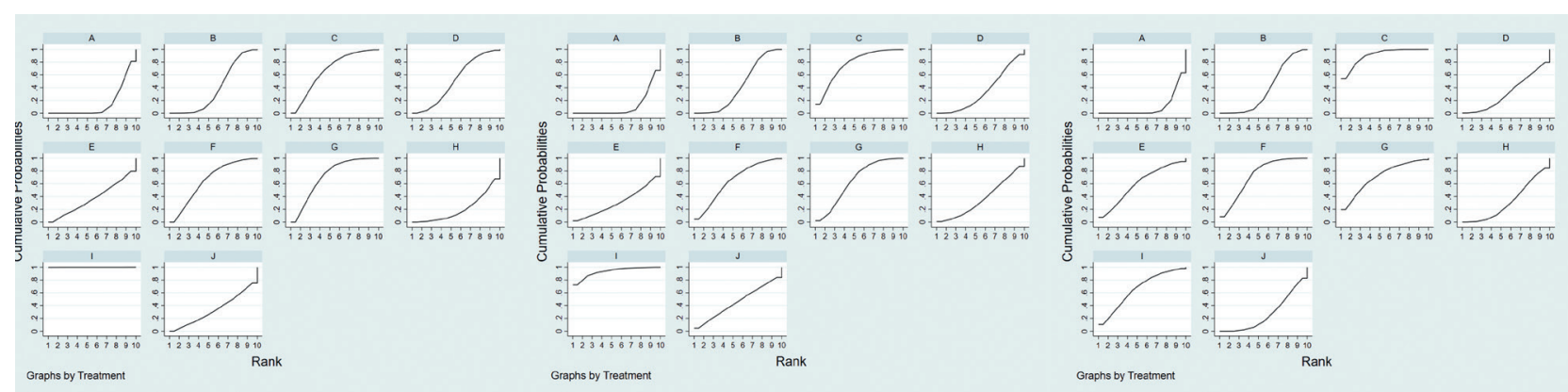

Fig. 5. Surface area under the cumulative ranking curve (SUCRA) of A: passive recovery (PAS); B: cold water immersion (CWI); C: the novel modality of cryotherapy (CRYO); D: cold pack; E: ice massage; F: phase change material (PCM); G: contrast water therapy (CWT); H: hot/warm water immersion (HWI/WWI); I: hot pack and J: ultrasound) treatment for the pain of patients with delayed onset muscle soreness (DOMS). Left: $24 \mathrm{~h}$ follow-up time; middle: $48 \mathrm{~h}$ follow-up time; right: $>48 \mathrm{~h}$ follow-up time.

after exercise. At a follow-up point of more than 48 $\mathrm{h}$ post-exercise, CRYO ranks first and shows the best analgesic effect. As a new cryotherapy, CRYO is similar to other traditional cold therapies. That is, by reducing muscle, skin and core temperature to stimulate cutaneous receptors and sympathetic adrenergic fibres, local blood vessels constrict, reducing local tissue metabolism and inflammation, and reducing the sensitivity of receptors and nerve conduction velocity, thus relieving the pain of patients with DOMS $(72,73)$. The pattern of intervention in CRYO involves exposure to extremely cold dry air $\left(<-100^{\circ} \mathrm{C}\right)$ in an environmentally controlled room for short periods of time, and patients are exposed to vaporized liquid nitrogen in a head-free cabin (41), or localized application of very cold air $\left(-30^{\circ} \mathrm{C}\right)$ on the skin and the subepidermal tissues by convection (8). Considering the extremely low temperature, the researchers only allowed patients to undergo CRYO for 2-5 min. However, this technique was found to be advantageous. A short period of application could reduce the intensity of pain and promote the recovery of DOMS through exposure to extremely cold air. Our results showed that CRYO was less effective than hot pack and CWT for analgesia within 48-h post-exercise, which may be because extremely low

Table VI. Ranking according to efficacy outcomes of surface under the cumulative ranking curve (SUCRA)

\begin{tabular}{|c|c|c|c|c|c|c|}
\hline \multirow[b]{2}{*}{ Intervention } & \multicolumn{2}{|l|}{$24 \mathrm{~h}$} & \multicolumn{2}{|l|}{$48 \mathrm{~h}$} & \multicolumn{2}{|l|}{$>48 \mathrm{~h}$} \\
\hline & SUCRA \% & $\begin{array}{l}\text { Mean } \\
\text { rank }\end{array}$ & SUCRA \% & $\begin{array}{l}\text { Mean } \\
\text { rank }\end{array}$ & SUCRA\% & $\begin{array}{l}\text { Mean } \\
\text { rank }\end{array}$ \\
\hline PAS & 15.1 & 8.6 & 11.2 & 9.0 & 9.8 & 9.1 \\
\hline CWI & 38.7 & 6.5 & 42.5 & 6.2 & 38.4 & 6.5 \\
\hline CRYO & 67.3 & 3.9 & 76.8 & 3.1 & 90.3 & 1.9 \\
\hline Cold pack & 51.1 & 5.4 & 34.1 & 6.9 & 32.3 & 7.1 \\
\hline Ice massage & 37.0 & 6.7 & 31.2 & 7.2 & 60.0 & 4.6 \\
\hline PCM & 65.3 & 4.1 & 64.7 & 4.2 & 72.9 & 3.4 \\
\hline CWT & 71.5 & 3.6 & 64.4 & 4.2 & 71.3 & 3.6 \\
\hline HWI/WWI & 20.6 & 8.1 & 36.5 & 6.7 & 32.2 & 7.1 \\
\hline Hot pack & 99.9 & 1.0 & 93.1 & 1.6 & 65.6 & 4.1 \\
\hline Ultrasound & 33.4 & 7.0 & 45.5 & 5.9 & 27.2 & 7.6 \\
\hline
\end{tabular}

SUCRA: surface under the cumulative ranking curve. PAS: passive recovery CWI: cold water immersion; CRYO: the novel modality of cryotherapy; PCM: phase change material; CWT: contrast water therapy; HWI/WWI: hot/warm water immersion. temperature stimulation did not provide a comfortable temperature for the patients when they received the cold therapy intervention. However, after the patients received cold therapy (more than $48 \mathrm{~h}$ post-exercise), the hypothermic effect of CRYO still affected the skin tissue, and the analgesic effect was gradually significant. CRYO may be a double-edged sword, as adverse reactions may occur due to its extremely low temperature. Although Banfi et al. (74) concluded that the new cryotherapy is safe and does not have a deleterious effect on immunological or cardiac function, there was some evidence that it is beneficial for muscle pain and harmful for the recovery of muscle function in a randomized controlled trial (RCT) (18). Therefore, future research should enhance the monitoring and reporting of adverse reactions, in order to improve the safety and effectiveness of the CRYO interventions.

Both PCM and CRYO belong to new type of cold therapy with the same mechanism of action. Our results showed that at the follow-up point of more than $48 \mathrm{~h}$ post-exercise, the cumulative area under the curve of PCM was the second largest value, indicating that the analgesic effect of PCM was second only to that of $\mathrm{CRYO}$ and superior to other interventions. analgesic effect on DOMS. PCM can prolong the duration of cryotherapy exposure while allowing the patient to continue with activities of daily living, which are absent from other interventions (75). PCM could not only maintain its own constant temperature, but also maintain the patient's skin temperature at $22^{\circ} \mathrm{C}$ during $3 \mathrm{~h}$ of application (57). One study (76) reported that PCM helped untrained individuals to recover from muscle injury and is more effective at alleviating the severity of pain in patients with DOMS. Although PCM has a good analgesic effect on patients with DOMS in our study, there were only 3 relevant studies, the number of studies and the sample size are small, and the first author of 2 studies is the same. In addition, the experimental design of the included original studies is limited. This may produce a placebo effect when participants receive PCM intervention, especially for 
subjective pain measurement. Therefore, more highquality studies are needed to confirm the analgesic effect of PCM.

At the 24-h post-exercise follow-up point, CWT ranked second only to hot pack in analgesic effect. In the included studies, CWT intervention time was relatively long (mean $14 \mathrm{~min}$ ). The mean water temperature of cold immersion in CWT was $12^{\circ} \mathrm{C}$, and that of hot immersion was between $38^{\circ} \mathrm{C}$ and $42^{\circ} \mathrm{C}$. CWT relieves pain in DOMS patients by producing hydrostatic pressure (77). And, the hydrostatic pressure is not related to the water temperature, but is related to the depth of immersion. In addition to the mechanisms of hydrotherapy, cold or heat therapy has a unique advantage. Benefits associated with CWT may be linked to changes in intra-muscular hydrostatic pressure by alternating vasoconstriction and vasodilation, which may alter blood flow in immersed musculature (78). CWT is associated with an increase in limb blood flow during warm immersion and a decrease during cold immersion (78). The alternate vasodilatation and vasoconstriction of the peripheral blood vessels has been proposed to increase lactate clearance, decrease oedema and increase blood flow $(20,79)$. These effects may play a positive role in relieving pain in patients with DOMS. However, at the follow-up point $24 \mathrm{~h}$ later, according to the area probability ranking under the curve, the analgesic effect of CWT decreases slightly, indicating that the effect is not sustained for a long time and the pain of DOMS may be relieved only temporarily. However, compared with the 2 new cold therapy methods (PCM and CRYO), the use of CWT may be relatively simple and safe. However, it should be noted that the intervention time and temperature of CWT in each study included were not completely the same, and the exercise training program for inducing DOMS has not been unified so far, so these factors may affect the accuracy of our results.

In summary, hot pack is better at reducing pain in patients with DOMS within $48 \mathrm{~h}$ follow-up times. CRYO is a primary choice for pain relief over $48 \mathrm{~h}$ post-exercise, and PCM is a suboptimal choice. This study showed that these latter 2 treatments may be good choices. However, hot pack is superior to CRYO and PCM with regard to safety and convenience. However, there are few reports on the application of the method in patients with DOMS. CRYO and PCM may have side-effects, so it is important to closely observe and monitor the changes in patients' vital signs and functions when applying this technology. Taking these factors into account, CWT may be considered a priority to alleviate the pain response caused by DOMS.

However, it is important to note that these recommendations should be treated with caution. The patient's sex, different exercise protocols, therapy intervention (time, temperature, frequency, etc.), and other factors may affect the stability of the results of this study. To date, there is no unified standard and regulation for the therapy intervention (time, frequency, temperature, etc.) of cold and heat therapy. Jinnah et al. (10) recommend use of cold pack for more than $10 \mathrm{~min}$ to relieve the pain of DOMS. For CWI, Jinnah et al. believe that soaking in cold water at $11-15^{\circ} \mathrm{C}$ for $11-15$ min is the most appropriate. In the included studies, most of the CWI intervention times were $10-15 \mathrm{~min}$, and the mean intervention temperature was $10-15^{\circ} \mathrm{C}$. This result seems to be consistent with previous research. In the included studies, the intervention time for cold pack was usually $20 \mathrm{~min}$. For the hot pack, 3 studies applied low-intensity hot pack for $8 \mathrm{~h}$, while the other study applied the hot pack for $30 \mathrm{~min}$. It is difficult to draw a clear conclusion on the differences in the intervention time. However, according to the characteristics of the included studies, we suggest that it is more appropriate to soak for $10-15 \mathrm{~min}$ in cold water at $10-15^{\circ} \mathrm{C}$ for the application of CWI, while, for hot pack, a longer intervention is recommended. However, one point needs to be made clear; when choosing any kind of intervention, it is important to fully evaluate the patient's physical condition, in order to select appropriate and effective intervention measures, and avoid the occurrence of frostbite or scald and other risks. Regarding sex, most of the included studies had only female, or only male, subjects. Therefore, sex may have influenced the results of the study. Hohenauer (12) conducted a subgroup analysis of the sexes, and found that men are more likely than women to benefit from cooling. According to Petrofsky (11), the thickness of fat in the area where DOMS occurs may be a factor influencing the effectiveness of heat or cold therapy. The thicker the subcutaneous fat, the slower the process of heat and cold therapy. However, fat thickness is not measured or analysed in any of the included studies. Therefore, whether sex differences and adipose tissue influence the intervention effect of heat or cold therapy requires further research, with more female participants. Regarding the change in intensity of the exercise programme, schemes for exercise-induced muscle injury vary from long sprints to competition. Subgroup analysis according to different exercise regimens was considered, but was not possible due to the wide range of exercise regimens included in the study. Therefore, future research is needed to determine a standard training programme to induce DOMS, in order to ensure stability of the results.

\section{Strengths and limitations}

This study has several strengths. The fundamental strength of the current analysis is the use of robust 
methodology. The comprehensive literature search was performed in 8 electronic databases, with an exhaustive search of articles and complementary sources. Secondly, to the best of our knowledge, this is the first network meta-analysis comparing the efficacy of different cold and heat therapies. The strengths of this systematic review relate mainly to the study interventions, which aim to rank the effectiveness of existing heat and cold therapy in reducing the pain of DOMS. However, this network meta-analysis has several limitations. First, the quality of the included studies varied. Some studies were better designed RCTs with adequate randomization; however, most studies had weak blinding/allocation. In trials with subjectively assessed outcomes, lack of adequate allocation, concealment, or blinding, tend to produce over-optimistic estimates of the effects of interventions (80). However, the pain score that needs to be analysed in this study belongs to the subjective evaluation result; thus, a lack of blinding would be expected to introduce bias if knowledge of the intervention groups affected the care received or the assessment of outcomes. Therefore, researchers should improve the transparency of the trial, and perform sufficient randomized allocation and complete blinding. Due the real-life situation, a relevant trial study of hot and cold therapy cannot achieve complete blinding, but a placebo control group could be included to avoid overstating the effects of an intervention. Secondly, in the included studies, the number of studies including some interventions was limited and the sample size was small, which may have affected the stability of the results resulting in publication bias and small sample size study effects. Finally, both publication and language restriction bias may have influenced the results.

\section{Conclusion}

This meta-analysis compared the effectiveness of 9 cold and heat modalities and 1 passive recovery (PAS) on pain relief on patients with DOMS. Regarding the 2 follow-up effectiveness time-points (within $48 \mathrm{~h}$ ), hot pack proved more effective and stable for pain relief compared with other interventions and is therefore a promising candidate for clinical application. If only the effectiveness of the intervention is considered, CRYO is a primary choice for pain relief over $48 \mathrm{~h}$ after exercise in our study, and PCM is a suboptimal choice. For professional athletes and any other subjects with DOMS, PCM and CRYO are helpful. But their side-effects and adverse reactions on the body have been less reported. Therefore, compared with CRYO and PCM, the use of CWT may be relatively simple and safe. In addition, due to the large inconsistencies in relevant studies on cold and hot pack included in this analysis, the effect of hot pack on reducing the degree of pain due to DOMS should be studied further in more detail. Further research and more high-quality RCTs are required to draw firm conclusions.

\section{ACKNOWLEDGEIMENTS}

The authors are grateful to the authors of the primary studies. This study was supported by National Nature Science Foundation of China (71704071), the fund of China Medical Board (\#20-374), Natural Science Foundation of Gansu Province (20JR10RA603), the Research Funds for the School of Nursing of Lanzhou University (LZUSON202002), the Fundamental Research Funds for the Central Universities (lzujbky-2021-33; lzujbky-2020-10), and Gansu Provincial Health Industry Research Program in 2017 (GSWSKY2017-73).

The authors have no conflicts of interest to declare.

\section{REFERENCES}

1. Armstrong RB. Mechanisms of exercise-induced delayed onset muscular soreness: a brief review. Med Sci Sports Exerc 1984; 16: 529-538.

2. Cleak MJ, Eston RG. Delayed onset muscle soreness: mechanisms and management. J Sports 1992; 10: 325-341.

3. Lightfoot TJ, Char D, McDermott J, Goya C. Immediate postexercise massage does not attenuate delayed onset muscle soreness. J Strength Cond Res 1997; 11: 119-124.

4. Cheung K, Hume P, Maxwell L. Delayed onset muscle soreness: treatment strategies and performance factors. Sports Med 2003; 33: 145-164.

5. Zhao L. Study on the pathogenesis and prevention of delayed muscle soreness. Wushu Studies 2017; 2: 136-139.

6. Connolly DA, Sayers SP, McHugh MP. Treatment and prevention of delayed onset muscle soreness. J Strength Cond Res 2003; 17: 197-208.

7. Malmir K, Ghotbi N, Mir SM, Moradi B. Comparing effects of cryotherapy and transcutaneous electrical nerve stimulation on signs and symptoms of delayed onset muscle soreness in amateur athletes. Open Pain J 2017; 10: 73-80.

8. Guilhem G, Hug F, Couturier A, Regnault S, Bournat L, Filliard JR, et al. Effects of air-pulsed cryotherapy on neuromuscular recovery subsequent to exercise-induced muscle damage. Am J Sports Med 2013; 41: 1942-1951.

9. Costello JT, Algar LA, Donnelly AE. Effects of whole-body cryotherapy ( -110 degrees $C)$ on proprioception and indices of muscle damage. Scand J Med Sci Sports 2012; 22: $190-198$.

10. Jinnah AH, Luo TD, Mendias C, Freehill M. Cryotherapy duration is critical in short-term recovery of athletes: a systematic review. J ISAKOS 2019; 4: 131-136.

11. Petrofsky JS, Khowailed IA, Lee H, Berk L, Bains GS, Akerkar $\mathrm{S}$, et al. Cold vs. heat after exercise - is there a clear winner for muscle soreness. J Strength Cond Res 2015; 29: 3245-3252.

12. Hohenauer E, Taeymans J, Baeyens JP, Clarys P, Clijsen $R$. The effect of post-exercise cryotherapy on recovery characteristics: a systematic review and meta-analysis. PLoS One 2015; 10: e0139028.

13. Hutton B, Salanti G, Caldwell DM, Chaimani A, Schmid $\mathrm{CH}$, Cameron $\mathrm{C}$, et al. The PRISMA extension statement for reporting of systematic reviews incorporating network meta-analyses of health care interventions: checklist and explanations. Ann Intern Med 2015; 162: 777-784.

14. Higgins JP, Altman DG. Assessing risk of bias in included studies. Cochrane handbook for systematic reviews of interventions. Cochrane book series 2008; 187-241. [Cited 2021 Jun 15]. Available from: https://doi. org/10.1002/9780470712184.ch8. 
15. Salanti G, Ades AE, Ioannidis JP. Graphical methods and numerical summaries for presenting results from multipletreatment meta-analysis: an overview and tutorial. J Clin Epidemiol 2011; 64: 163-171.

16. Yanagisawa O, Niitsu M, Yoshioka H, Goto K, Kudo H, Itai Y. The use of magnetic resonance imaging to evaluate the effects of cooling on skeletal muscle after strenuous exercise. Eur J Appl Physiol 2003; 89: 53-62.

17. Wilson LJ, Dimitriou L, Hills FA, Gondek MB, Cockburn E. Whole body cryotherapy, cold water immersion, or a placebo following resistance exercise: a case of mind over matter? . Eur J Appl Physiol 2019; 119: 135-147.

18. Wilson LJ, Cockburn E, Paice K, Sinclair S, Faki T, Hills FA, et al. Recovery following a marathon: a comparison of cold water immersion, whole body cryotherapy and a placebo control. Eur J Appl Physiol 2017; 118: 153-163.

19. Vieira A, Siqueira AF, Ferreira-Junior JB, do Carmo J, Durigan $J L$, Blazevich $A$, et al. The effect of water temperature during cold-water immersion on recovery from exercise-induced muscle damage. Int J Sports Med 2016; 37: 937-943.

20. Vaile JM, Gill ND, Blazevich AJ. The effect of contrast water therapy on symptoms of delayed onset muscle soreness. J Strength Cond Res 2007; 21: 697-702.

21. Vaile J, Halson S, Gill N, Dawson B. Effect of hydrotherapy on the signs and symptoms of delayed onset muscle soreness. Eur J Appl Physiol 2008; 102: 447-455.

22. Skurvydas A, Sipaviciene S, Krutulyte G, Gailiuniene A, Stasiulis A, Mamkus G, et al. Cooling leg muscles affects dynamics of indirect indicators of skeletal muscle damage. J Back Musculoskelet Rehabil 2006; 19: 141-151.

23. Siqueira AF, Vieira A, Bottaro M, Ferreira-Junior JB, Nobrega OT, de Souza VC, et al. Multiple cold-water immersions attenuate muscle damage but not alter systemic inflammation and muscle function recovery: a parallel randomized controlled trial. Sci Rep 2018; 8: 10961.

24. Sellwood KL, Brukner $P$, Williams D, Nicol A, Hinman R. Ice-water immersion and delayed-onset muscle soreness: a randomised controlled trial. Br J Sports Med 2007; 41: 392-397.

25. Pointon $M$, Duffield R, Cannon J, Marino FE. Cold application for neuromuscular recovery following intense lower-body exercise. Eur J Appl Physiol 2011; 111: 2977-2986.

26. Petrofsky JS, Laymon M, Berk L, Al-Nakhli HH, Banh A, Eisentrout $A$, et al. Pilot study: physiological evidence that heat reduces pain and muscle damage in delayed-onset muscle soreness. Clin Pract 2012; 9: 639-650.

27. Petrofsky J, Berk L, Bains G, Khowailed IA, Lee H, Laymon $M$. The efficacy of sustained heat treatment on delayed-onset muscle soreness. Clin J Sport Med 2017; 27: 329-337.

28. Naugle KE, Parr JJ, Chang S, Naugle KM. Active gaming as pain relief following induced muscle soreness in a college-aged population. Athl Train Sports Health Care 2017; 9: 225-232.

29. Machado AF, Almeida AC, Micheletti JK, Vanderlei FM, Tribst MF, Netto Junior J, et al. Dosages of cold-water immersion post exercise on functional and clinical responses: a randomized controlled trial. Scand J Med Sci Sports 2017; 27: $1356-1363$.

30. Lindsay A, Carr S, Cross S, Petersen C, Lewis JG, Gieseg SP. The physiological response to cold-water immersion following a mixed martial arts training session. Appl Physiol Nutr Metab 2017; 42: 529-536.

31. Leeder JD, van Someren KA, Bell PG, Spence JR, Jewell AP, Gaze D, et al. Effects of seated and standing cold water immersion on recovery from repeated sprinting. J Sports Sci 2015; 33: 1544-1552.

32. Kwiecien SY, O'Hara DJ, McHugh MP, Howatson G. Prolonged cooling with phase change material enhances recovery and does not affect the subsequent repeated bout effect following exercise. Eur J Appl Physiol 2020; 120: 413-423.

33. Kwiecien SY, McHugh MP, Howatson G. The efficacy of cooling with phase change material for the treatment of exercise-induced muscle damage: pilot study. J Sports Sci 2018; 36: 407-413.

34. Kuligowski LA, Lephart SM, Giannantonio FP, Blanc RO Effect of whirlpool therapy on the signs and symptoms of delayed-onset muscle soreness. J Athl Train 1998; 33: 222-228.

35. Jakeman JR, Macrae R, Eston R. A single 10-min bout of cold-water immersion therapy after strenuous plyometric exercise has no beneficial effect on recovery from the symptoms of exercise-induced muscle damage. Ergonomics 2009; 52: 456-460.

36. Crowther F, Sealey R, Crowe M, Edwards A, Halson S. Influence of recovery strategies upon performance and perceptions following fatiguing exercise: a randomized controlled trial. BMC Sports Sci Med Rehabil. 2017; 9: 25.

37. Ingram J, Dawson B, Goodman C, Wallman K, Beilby J. Effect of water immersion methods on post-exercise recovery from simulated team sport exercise. J Sci Med Sport 2009; 12: 417-421.

38. Howatson G, Van Someren KA. Ice massage. Effects on exercise-induced muscle damage. J Sports Med Phys Fitness 2003; 43: 500-505.

39. Howatson G, Gaze D, van Someren KA. The efficacy of ice massage in the treatment of exercise-induced muscle damage. Scand J Med Sci Sports 2005; 15: 416-422.

40. Hohenauer E, Costello JT, Stoop R, Kung UM, Clarys P, Deliens $\mathrm{T}$, et al. Cold-water or partial-body cryotherapy? Comparison of physiological responses and recovery following muscle damage. Scand J Med Sci Sports 2018; 28: 1252-1262.

41. Hohenauer E, Costello JT, Deliens T, Clarys P, Stoop R, Clijsen R. Partial-body cryotherapy ( -135 degrees $C$ ) and cold-water immersion (10 degrees C) after muscle damage in females. Scand J Med Sci Sports 2020; 30: 485-495.

42. Higgins TR, Climstein M, Cameron M. Evaluation of hydrotherapy, using passive tests and power tests, for recovery across a cyclic week of competitive rugby union. J Strength Cond Res 2013; 27: 954-965.

43. Glasgow PD, Ferris R, Bleakley CM. Cold water immersion in the management of delayed-onset muscle soreness: is dose important? A randomised controlled trial. Phys Ther Sport 2014; 15: 228-233.

44. French DN, Thompson KG, Garland SW, Barnes CA, Portas $M D$, Hood PE, et al. The effects of contrast bathing and compression therapy on muscular performance. Med Sci Sports Exerc 2008; 40: 1297-1306.

45. Fonseca LB, Brito CJ, Silva RJ, Silva-Grigoletto ME, da Silva WMJ, Franchini E. Use of Cold-water immersion to reduce muscle damage and delayed-onset muscle soreness and preserve muscle power in jiu-jitsu athletes. J Athl Train 2016; 51: 540-549.

46. Fonda B, Sarabon N. Effects of whole-body cryotherapy on recovery after hamstring damaging exercise: a crossover study. Scand J Med Sci Sports 2013; 23: e270-278.

47. Ferreira-Junior JB, Bottaro M, Vieira A, Siqueira AF, Vieira CA, Durigan JL, et al. One session of partial-body cryotherapy ( -110 degrees $C$ ) improves muscle damage recovery. Scand J Med Sci Sports 2015; 25: e524-530.

48. Eston R, Peters D. Effects of cold water immersion on the symptoms of exercise-induced muscle damage. J Sports Sci 1999; 17: 231-238.

49. Elias GP, Wyckelsma VL, Varley MC, McKenna MJ, Aughey RJ. Effectiveness of water immersion on postmatch recovery in elite professional footballers. Int J Sport Physiol Perform 2013; 8: 243-253.

50. Elias GP, Varley MC, Wyckelsma VL, McKenna MJ, Minahan $\mathrm{CL}$, Aughey RJ. Effects of water immersion on posttraining recovery in Australian footballers. Int J Sport Physiol Perform 2012; 7: 357-366.

51. Doungkulsa A, Paungmali A, Henry Joseph L, Khamwong P. Effectiveness of air pulsed cryotherapy on delayed onset muscle soreness of elbow flexors following eccentric exercise. Polish Annals Med 2018; 25: 103-111. 
52. Doeringer JR, Colas M, Peacock C, Gatens DR. The effects of postexercise cooling on muscle performance and soreness perception. Athl Ther Today 2018; 23: 73-76.

53. de Paiva PR, Tomazoni SS, Johnson DS, Vanin AA, Albuquerque-Pontes GM, Machado $C D$, et al. Photobiomodulation therapy (PBMT) and/or cryotherapy in skeletal muscle restitution, what is better? A randomized, double-blinded, placebo-controlled clinical trial. Laser Med Sci 2016; 31 : 1925-1933.

54. de Freitas VH, Ramos SP, Bara-Filho MG, Freitas DGS, Coimbra DR, Cecchini R, et al. Effect of cold water immersion performed on successive days on physical performance, muscle damage, and inflammatory, hormonal, and oxidative stress markers in volleyball players. J Strength Cond Res 2019; 33: 502-513.

55. Dantas G, Barros A, Silva B, Belém L, Ferreira V, Fonseca $A$, et al. Cold-water immersion does not accelerate performance recovery after 10-km street run: randomized controlled clinical trial. Res Q Exerc Sport 2019; 91: 228-238.

56. Crystal NJ, Townson DH, Cook SB, LaRoche DP. Effect of cryotherapy on muscle recovery and inflammation following a bout of damaging exercise. Eur J Appl Physiol 2013; 113: 2577-2586.

57. Clifford T, Abbott W, Kwiecien SY, Howatson G, McHugh MP. Cryotherapy reinvented: application of phase change material for recovery in elite soccer. Int J Sport Physiol Perform 2018; 13: 584-589.

58. Ciccone CD, Leggin BG, Callamaro JJ. Effects of ultrasound and trolamine salicylate phonophoresis on delayed-onset muscle soreness. Phys Ther 1991; 71: 666-675.

59. Brukner P, Sellwood K, Williams D, Nicol A, Hinman R. Ice water immersion and delayed onset muscle soreness: a randomised controlled trial. Med Sci Sports Exerc 2005; 37: S276-S276.

60. Bouzid MA, Ghattassi K, Daab W, Zarzissi S, Bouchiba M, Masmoudi $L$, et al. Faster physical performance recovery with cold water immersion is not related to lower muscle damage level in professional soccer players. J Therm Biol 2018; 78: 184-191.

61. Behringer M, Jedlicka D, Mester J. Effects of Iymphatic drainage and cryotherapy on indirect markers of muscle damage. J Sports Med Phys Fitness 2018; 58: 903-909.

62. Bailey DM, Erith SJ, Griffin PJ, Dowson A, Brewer DS, Gant $\mathrm{N}$, et al. Influence of cold-water immersion on indices of muscle damage following prolonged intermittent shuttle running. J Sports Sci 2007; 25: 1163-1170.

63. Aytar A, Tuzun EH, Eker L, Yuruk ZOB, Daskapan A, Akman MN. Effectiveness of low-dose pulsed ultrasound for treatment of delayed-onset muscle soreness: a doubleblind randomized controlled trial. Isokinet Exerc Sci 2008; 16: 239-247.

64. Ascensao A, Leite M, Rebelo AN, Magalhaes S, Magalhaes J. Effects of cold water immersion on the recovery of physical performance and muscle damage following a one-off soccer match. J Sports Sci 2011; 29: 217-225.

65. Adamczyk JG, Krasowska I, Boguszewski D, Reaburn P. The use of thermal imaging to assess the effectiveness of ice massage and cold-water immersion as methods for supporting post-exercise recovery. J Therm Biol 2016; 60: $20-25$.

66. Abaidia AE, Lamblin J, Delecroix B, Leduc C, McCall A Nedelec $M$, et al. Recovery from exercise-induced muscle damage: cold-water immersion versus whole-body cryotherapy. Int J Sports Physiol Perform 2017; 12: 402-409.

67. Hausswirth C, Louis J, Bieuzen F, Pournot H, Fournier J, Filliard JR, et al. Effects of whole-body cryotherapy vs. farinfrared vs. passive modalities on recovery from exerciseinduced muscle damage in highly-trained runners. PLoS One 2011; 6: e277749.

68. Barber S, Pattison J, Brown F, Hill J. Efficacy of repeated cold water immersion on recovery after a simulated rugby union protocol. J Strength Cond Res 2020; 34: 3523-3529.

69. Kositsky A, Avela J. The effects of cold water immersion on the recovery of drop jump performance and mechanics: a pilot study in under-20 soccer players. Front Sports Act Living 2020; 2: 17.

70. Dantas G, Barros A, Silva B, Belém L, Ferreira V, Fonseca $A$, et al. Cold water immersion does not accelerate performance recovery after $10-\mathrm{km}$ street run. Res Q Exerc Sport 2020; 91 : 228-238.

71. French SD, Cameron M, Walker BF, Esterman AJ. Superficial heat or cold for low back pain. Cochrane Database Syst Rev 2006: CD004750.

72. Costello JT, Baker PR, Minett GM, Bieuzen F, Stewart IB, Bleakley C. Cochrane review: whole-body cryotherapy (extreme cold air exposure) for preventing and treating muscle soreness after exercise in adults. J Evid Based Med 2016; 9: 43-44.

73. Bleakley C, McDonough S, Gardner E, Baxter GD, Hopkins JT, Davison GW. Cold-water immersion (cryotherapy) for preventing and treating muscle soreness after exercise. Cochrane Database Syst Rev 2012: CD008262.

74. Banfi G, Lombardi G, Colombini A, Melegati G. Whole-body cryotherapy in athletes. Sports Med 2010; 40: 509-517.

75. Reinertsen RE, Faerevik H, Holbo K. Optimizing the performance of phase-change materials in personal protective clothing systems. Int J Occup Saf Ergon 2008; 14: 43-53.

76. Kwiecien SY, McHugh MP, Howatson G. The efficacy of cooling with phase change material for the treatment of exercise-induced muscle damage: pilot study. J Sport Sci 2018; 36: 407-413.

77. Higgins TR, Greene DA, Baker MK. Effects of cold water immersion and contrast water therapy for recovery from team sport: a systematic review and meta-analysis. J Strength Cond Res 2017; 31: 1443-1460.

78. Bieuzen F, Bleakley CM, Costello JT. Contrast water therapy and exercise induced muscle damage: a systematic review and meta-analysis. PLoS One 2013; 8: e62356.

79. Cochrane DJ. Alternating hot and cold water immersion for athlete recovery: a review. Phys Ther Sport 2004; 5 : 26-32.

80. Wood L, Egger M, Gluud LL, Schulz KF, Juni P, Altman $D G$, et al. Empirical evidence of bias in treatment effect estimates in controlled trials with different interventions and outcomes: meta-epidemiological study. BMJ 2008; 336: 601-605. 